I N S T I T U T O

$\mathrm{DE}$

M E D I C I N A

T R O P I C A L

DE

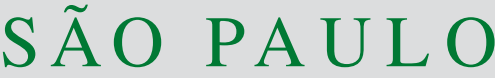

JOURNAL OF THE SÃO PAULO INSTITUTE OF TROPICAL MEDICINE

${ }^{1}$ Community Health Center Zvezdara, Belgrade, Serbia

${ }^{2}$ University of Belgrade, Faculty of Medicine, Internal Medicine Department, Belgrade, Serbia

${ }^{3}$ University Clinical Center of Serbia, Teaching Hospital of Pulmonology, Belgrade, Serbia

${ }^{4}$ Public Health Institute of Serbia "Dr Milan Jovanović Batut," Department of HIV, Hepatitis, STDs and TB, Belgrade, Serbia

Correspondence to: Dragica Petar Pesut University of Belgrade, Faculty of Medicine, Suboticeva 8, 11000, Beograd, Serbia Tel: +381 113663956

Fax: +381113615561

E-mail: dragica.pesut@gmail.com

Received: 26 May 2021

Accepted: 2 June 2021

\section{Influence of the COVID-19 pandemic on the incidence of tuberculosis and influenza}

\author{
Jovana Milan Pavlovic ${ }^{\circledR}$, Dragica Petar Pesut ${ }^{(2,3}$, Maja Borivoje Stosic ${ }^{\circledR}$
}

\section{ABSTRACT}

We examined whether the COVID-19 pandemic has affected the incidence of tuberculosis (TB) and influenza in Serbia, a Southeast European country with a low TB incidence rate and a mandatory BCG vaccination at birth. The first case of COVID-19 was registered on March 6, 2020. Despite the need for a sudden adaptation of the health care system, routines of mycobacterial laboratories have never stopped. In 2020, the number of newly diagnosed TB patients was significantly lower than expected $(\mathrm{p}=0.04)$, but the number of patients with influenza increased when compared to 2019. Although many patients with influenza A H1N1 were observed before the beginning of the COVID-19 pandemic, the increment of cases could also be a consequence of cases of influenza with COVID-like symptoms detected thereafter. It may also be attributed to misclassification of clinical cases that were negative for SARS-CoV-2 and reported as influenza. Difficulties to seek medical attention because of the COVID-19 pandemic and possible underreporting are considered as reasons for the decline in the incidence rate of TB. On the other hand, individual and social measures to prevent the spread of SARS-CoV-2 such as wearing face masks, social distancing, lockdown, which were strictly applied to COVID-19 patients, health care staffs and most of the population, could have hindered TB infections more than the two viral diseases, which appear to be more contagious. The increased motivation of the population to protect their health during the COVID-19 pandemic provided an opportunity for their effective education. This is crucial in further combating TB as a preventable disease.

KEYWORDS: COVID-19. Tuberculosis. Influenza. Incidence.

\section{INTRODUCTION}

COVID-19, an infectious disease caused by the Severe Acute Respiratory Syndrome Coronavirus 2 (SARS-CoV-2) caused a pandemic that began in Wuhan, China, in 2019. The number of infected people in the world is constantly growing. By May 2021 there were 3,198,528 deaths among more than 152 million infected people worldwide ${ }^{1}$.

SARS-CoV-2 primarily affects the respiratory system and the most common symptoms are dry cough, fever, fatigue and shortness of breathe. Some patients may experience headache, sore throat, myalgia, diarrhea, vomiting, skin rash and loss of taste or smell (anosmia) ${ }^{2}$. This RNA virus uses ACE 2 receptors to enter the host cell. These receptors are expressed in lung alveoli, heart, central nervous system, kidney, gut and endothelial cells. In these locations, the virus causes extensive damage.

There is a wide spectrum of clinical presentations varying from asymptomatic infection and mild flu-like disease to serious life-threatening conditions with severe pneumonia, Acute Respiratory Distress Syndrome (ARDS) and sepsis ${ }^{2}$. In the central 
nervous system, macrovascular stroke and Guillain-Barré syndrome may develop ${ }^{3}$ and devastating complications may lead to neurological sequellae ${ }^{4}$. Some patients have acute kidney injury and conjunctivitis. SARS-CoV-2 compromises endothelial cells causing microthrombosis and occlusion $^{5}$. The changes in coagulation are reponsible for many COVID-19-related deaths. Despite a better understanding of COVID-19 that gained strength over time, there is still no definitive treatment protocol and the therapy is primarily supportive. Corticosteroids, anticoagulants and endothelial stabilizers are very important drugs, together with antiviral agents, monoclonal antibodies, oligonucleotides, peptides and interferons, used against the hyperinflammation state that characterizes COVID-19. The real solution to mitigate the disease is a suitable vaccine ${ }^{6}$.

Before the COVID-19 pandemic, tuberculosis (TB), an infectious disease caused by Mycobacterium tuberculosis complex bacteria, was a top cause of death worldwide, especially in underdeveloped countries, which are also the most affected by COVID-19. TB was responsible for 1.4 million deaths in $2019^{7}$. One third of the world's population is infected by $M$. tuberculosis, i.e., they suffer from latent TB infection (LTBI). The risk of active TB disease in such populations is $5-10 \%$. General symptoms of TB (fever, night sweats, weight loss) precede the onset of respiratory symptoms (cough, dyspnea, chest pain), so that TB can go unnoticed for months, resulting in delayed diagnoses and further transmission of bacilli to others in the community.

In some patients with synchronous active TB and COVID-19, the diagnosis of the latter was established before that of tuberculosis ${ }^{8}$. Although the signs and symptoms of COVID-19 and TB can be very similar, the SARS-CoV-2 infection causes acute symptoms with sudden high fever, cough and fatigue that make individuals seek medical attention earlier. The high index of suspicion during the COVID-19 pandemic has also contributed to faster diagnoses.

Some infectious diseases like measles and whooping cough can seriously disrupt the human immunity, especially during the recovery period. It is possible that COVID-19 can trigger the transition from LTBI to active TB in a patient, but the potential contribution of COVID-19 for the development of active TB disease remains unrevealed. The influence of a preexisting TB on the onset of severe COVID-19-related pneumonia and on the disease outcome should be further studied with a larger sample size. The mortality rate of the coinfection is $12.3 \%$, which is significantly higher than for COVID-19 alone ${ }^{9}$. The patients at greatest risk are those with comorbidities and/or an impaired immune response, such as those with HIV coinfection, malignant diseases, immunodeficiencies and old age. Other common risk factors for the two infectious diseases are malnutrition, smoking and alcohol abuse $\mathrm{e}^{10,11}$.

Therefore, it is likely that COVID-19 may worsen the epidemiological situation of tuberculosis, at least in TB endemic areas where poverty greatly contributes to the $\mathrm{TB}$ prevalences ${ }^{11}$. The aim of this study was to investigate how the COVID-19 pandemic has affected the incidence of TB in Serbia during 2020.

\section{MATERIALS AND METHODS}

Serbia lies at the crossroads of Central and Southeast Europe between latitudes $41^{\circ}$ and $47^{\circ} \mathrm{N}$, and longitudes $18^{\circ}$ and $23^{\circ} \mathrm{E}$, on the Balkan peninsula. The country went through an economic crisis during the 1990s, when a stable TB trend took place, instead of the continuous decrease that occurred previously. Moreover, the socio-economic transition period at the beginning of the $21^{\text {st }}$ century showed a gradually decreasing trend of the total TB incidence rate $^{7,12}$. The current rate of $9 / 100,000$ inhabitants places Serbia as a low TB incidence country ${ }^{7,13}$. BCG vaccination is mandatory at birth.

The first case of COVID-19 in Serbia was detected on March 6, 2020 followed by a rapid increase in the number of cases thereafter. The outbreak is still ongoing. The total number of cases confirmed by the middle of May 2021 was 707,185 with 6,646 deaths among the total population of $6,945,235$ inhabitants. The mortality rate is $0.95 \%{ }^{14}$. Protective measures such as face mask wearing (indoors and outdoors), social distancing and lockdown were all implemented to stop the spreading of the disease. Vaccination against COVID-19 started on $24^{\text {th }}$ December, 2020. About $40 \%$ of the adult population had been vaccinated by May $15^{\text {th }} 2021$. Despite an immediate adaptation of the health care system to respond to the COVID-19 pandemic, the routines of laboratories dedicated to mycobacterial diagnoses has never been interrupted.

The following laboratory methods are used in Serbia to define cases of COVID-19: Real-Time Polymerase Chain Reaction (RT-PCR) and the Rapid Antigen Test in nasopharyngeal secretions, together with serological tests for anti-SARS-CoV-2 IgM and IgG detection. RT PCR-negative patients with clinical and radiological signs suggestive of COVID-19 are also classified as COVID-19 without viral confirmation according to U07.2 from the International Classification. Concerning tuberculosis, the sputum smear for acid fast bacilli, Lowenstein-Jensen medium for culturing M. tuberculosis (gold standard), and molecular techniques for the identification of mycobacteria, including the GeneXpert MTB/RIF (Cepheid Sunnyvale, CA, United States) are available. Diagnosis of influenza 
is performed by RT-PCR of nasopharyngeal swabs and serological tests.

To meet the aim of the study, we used the Annual Report on Infectious Diseases of the Republic of Serbia for 2018 and 2019 issued by the Institute of Public Health of Serbia "Dr Milan Jovanović Batut" in Belgrade, preliminary data for 2020 with special regard to TB and influenza as well as the Central TB registry. The population surveillance of influenza in the 2020/2021 season started on September 28, $2020\left(40^{\text {th }}\right.$ reporting week to October $\left.4^{\text {th }} 2020\right)$ with weekly monitoring and through summary reports of influenza into the $20^{\text {th }}$ week of the following year. Data of this study were collected for the first 16 epidemiological weeks of the year 2021 and therefore were noted as partial. We applied the chi-square test to compare observed and expected values of incidence rates.

\section{RESULTS}

We found a further decline in the incidence rate of TB in Serbia, in 2020. The chi-square test showed that the value of 4.5/100.000 inhabitants is significantly lower than expected when compared to the trend from previous years $(p=0.04)$. At the same time, the number of detected and notified cases of influenza has increased when compared to the previous year (Table 1).

\section{DISCUSSION}

A similar situation of decreased TB incidence has been reported in some other countries. After the onset of the COVID-19 pandemic, the number of new cases of TB in South Korea decreased by $23-25 \%{ }^{15}$. Many years before the COVID-19 pandemic, both Brazil and India faced problems with TB control, having some of the highest burdens of TB correlated with poverty ${ }^{16}$. In $2019,450.000$ TB patients in India died, more than 1,000 people daily. In April 2020, during the COVID-19 pandemic, the number of TB patients was half that reported in February of the same year ${ }^{17}$. In China, the number of new TB cases in February 2020 was $20 \%$ lower than that of February $2019^{18}$. The situation is similar in Malaysia and in the Philippines ${ }^{19}$.

The decreased TB incidence rate that we found in 2020 could be explained by two main causes: difficulties of potentially preexisting TB patients to seek medical attention due to the situation of the COVID-19 pandemic, and possible underreporting of TB cases for the same reason. The need for a sudden reorganization of the health care system and the fear of contracting COVID-19 in health care facilities might have led some TB patients to prefer staying at home ${ }^{17}$. However, another rational explanation for such an impressive decrease of the TB incidence rate in Serbia may be related to the proper implementation of measures for the prevention of SARS-CoV-2 transmission that could have simultaneously contributed to prevent the transmission of other airborne infections.

On the other hand, the increase of the influenza incidence rate may indicate a better detection due to influenza cases that would not have sought medical attention during the previous years, but did so when they presented with COVID-19-like symptoms and as a consequence were detected and registered. In addition, the increase of the influenza incidence rate may be also attributed to the misclassification of SARS-CoV-2 cases that were RT-PCRnegative despite the clinical signs and symptoms suggestive of COVID-19, that may have been reported as influenza. Rare cases of coinfection COVID-19 and influenza A has also been seen in clinical practice.

Individual measures against the spread of airborne infections have been known for a long time in Serbia, but have never been applied as strictly as during the COVID-19 pandemic among all patients, health care staffs and most of the general population. They might have been able to stop TB more than COVID-19 and influenza, as COVID-19 and

Table 1 - Incidence of tuberculosis and influenza in Serbia, 2014-2020.

\begin{tabular}{lccccc}
\hline & \multicolumn{2}{c}{ TUBERCULOSIS } & & \multicolumn{2}{c}{ INFLUENZA } \\
\cline { 2 - 3 } \cline { 5 - 6 } Year & Number of patients & $\begin{array}{c}\text { Incidence rate } \\
(\mathrm{N} / 100,000 \text { Inhabitants })\end{array}$ & & Number of patients & $\begin{array}{c}\text { Incidence rate } \\
(\text { N/100,000inhabitants) }\end{array}$ \\
\hline 2014 & 1,083 & 15.1 & 13.5 & 24,973 & 348.6 \\
2015 & 962 & 11.8 & 40,845 & 572.7 \\
2016 & 837 & 10.5 & 44,131 & 622.0 \\
2017 & 743 & 9.9 & 33,787 & 478.7 \\
2018 & 695 & 9.0 & 171,901 & $2,388.0$ \\
2019 & 623 & $4.5^{*}$ & 41,072 & 591.4 \\
2020 & 302 & & 53,237 (partial data) & 766.6 \\
\hline
\end{tabular}

${ }^{\star}$ The tuberculosis incidence rate in 2020 was significantly lower than expected $(p=0.04)$. 
influenza seems to be more contagious than $\mathrm{TB}^{20}$. A similar observation about the influence of COVID-19 on TB control as a double-edged sword has been reported in China ${ }^{18}$.

Apart from COVID-19, TB remains a leading cause of mortality among infectious diseases in the world. Preventive measures against COVID-19 have been shown to be useful in the prevention of TB transmission as well. We have highlighted once more the importance of preventive measures in TB control, especially individual ones concerning cough hygiene and measures of environmental control, especially in health care facilities. The COVID-19 pandemic has enhanced the motivation for health protection among populations and offers a great opportunity for the implementation of educational measures aimed at preventing and controlling TB. The example of Serbia is illustrative in these terms. The lessons we learned from the COVID-19 pandemic may be useful in further controlling TB as a preventable disease.

\section{AUTHORS' CONTRIBUTIONS}

DP concepted the idea, all the authors analyzed the data, JP drafted the article together with DP and MS, and all the authors accepted the final version of the manuscript to be submitted for publication.

\section{CONFLICT OF INTERESTS}

The authors declare no conflict of interests.

\section{REFERENCES}

1. World Health Organization. Weekly operational update on COVID-19: 3 May 2021. [cited 2021 Jun 5]. Available from: https://www.who.int/publications/m/item/weekly-operationalupdate-on-covid-10---3-may-2021

2. Adil MT, Rahman R, Whitelaw D, Jain V, Al-Taan O, Rashid F, et al. SARS-CoV-2 and the pandemic of COVID-19. Postgrad Med J. 2021;97:110-6.

3. Scheidl E, Canseco DD, Hadji-Naumov A, Bereznai B. GuillainBarré syndrome during SARS-CoV-2 pandemic: a case report and review of recent literature. J Peripher Nerv Syst. 2020;25:204-7.

4. Orsini A, Corsi M, Santangelo A, Riva A, Peroni D, Foiadelli T, et al. Challenges and management of neurological and psychiatric manifestations in SARS-CoV-2 (COVID-19) patients. Neurol Sci. 2020;41:2353-66.

5. Hanff TC, Mohareb AM, Giri J, Cohen JB, Chirinos JA. Thrombosis in COVID-19. Am J Hematol. 2020;95:1578-89.

6. Menzella F, Matucci A, Vultaggio A, Barbieri C, Biava M, Scelfo $\mathrm{C}$, et al. COVID-19: general overview, pharmacological options and ventilatory support. Multidiscip Respir Med. 2020;15:708.
7. World Health Organization. Global tuberculosis report 2019. Geneva: WHO; 2019. [cited 2021 Jun 5]. Available from: https://www.who.int/teams/global-tuberculosis-programme/ tb-reports/global-report-2019

8. Migliori GB, Thong PM, Akkerman O, Alffenaar JW, ÁlvarezNavascués F, Assao-Neino MM, et al. Worldwide effects of Coronavirus disease pandemic on tuberculosis services, January-April 2020. Emerg Infect Dis. 2020;26:2709-12.

9. Tadolini M, Codecasa LR, García-García JM, Blanc FX, Borisov S, Alffenaar JW, et al. Active tuberculosis, sequelae and COVID-19 co-infection: first cohort of 49 cases. Eur Respir J. 2020;56:2001398.

10. Saunders MJ, Evans CA. COVID-19, tuberculosis and poverty: preventing a perfect storm. Eur Respir J. 2020;56:2001348.

11. Tadolini M, García-García JM, Blanc FX, Borisov S, Goletti D, Motta I, et al. On tuberculosis and COVID-19 co-infection. Eur Respir J. 2020;56:2002328.

12. Gledovic Z, Vlajinac H, Pekmezovic T, Grujicic-Sipetic S, Grgurevic A, Pesut D. Burden of tuberculosis: Serbian perspectives. In: Preedy VR, Watson RR, editors. Handbook of disease burdens and quality of life measures. New York: Springer; 2010. p.1211-20.

13. Serbia. Institute of Public Health "Dr Milan Jovanović Batut". Annual report on infectious diseases in Republic of Serbia in 2018. Belgrade: Institute of Public Health of Serbia "Dr Milan Jovanović Batut"; 2019. [cited 2021 Jun 5]. Available from: http://www.batut.org.rs/download/izvestaji/ GodisnjiIzvestajOZaraznimBolestima2018.pdf

14. Serbia. Ministry of Health. Institute for Public Health. Latest information about COVID-19 in the Republic of Serbia. [cited 2021 Jun 5]. Available from: https://covid19.rs/homepageenglish/

15. Kwak N, Hwang SS, Yim JJ. Effect of COVID-19 on tuberculosis notification, South Korea. Emerg Infect Dis. 2020;26:2506-8.

16. Ribeiro VS, Telles JP, Tuon FF. Concerns about COVID-19 and tuberculosis in Brazil: social and public health impacts. Enferm Infecc Microbiol Clin (Engl Ed). 2021;39:216-7.

17. Behera D. TB control in India in the COVID era. Indian J Tuberc. 2021;68:128-33.

18. Chen H, Zhang K. Insight into the impact of the COVID-19 epidemic on tuberculosis burden in China. Eur Respir J. 2020;56:2002710.

19. Chiang CY, Islam T, Xu C, Chinnayah T, Garfin AM, Rahevar $\mathrm{K}$, et al. The impact of COVID-19 and the restoration of tuberculosis services in the Western Pacific Region. Eur Respir J. 2020;56:2003054.

20. Zemouri C, Awad SF, Volgenant CM, Crielaard W, Laheij AM, de Soet JJ. Modeling of the transmission of coronaviruses, measles virus, influenza virus, Mycobacterium tuberculosis, and Legionella pneumophila in dental clinics. J Dent Res. 2020;99:1192-8. 